\section{P729 TREPONEMA PALLIDUM-PLATELET INTERACTIONS AND RELEVANCE TO TREPONEMAL INVASION}

Brigette Church*, Erika Wall, Caroline Cameron. University of Victoria, Biochemistry and Microbiology, Victoria, Canada

10.1136/sextrans-2019-sti.790

Background Treponema pallidum ssp. pallidum (Tp), the causative agent of syphilis, is an invasive pathogen that interacts with host cells during infection. In the bloodstream $T p$ encounters platelets, which are sentinel cells that activate and release specific components to enhance immune cell targeting to the site of infection/inflammation. Tumor cells and invasive pathogenic bacteria can associate with platelets and recognize these same secreted components, to allow for enhanced spread via the bloodstream. We have previously established that $T p$ interacts with platelets: in this study we probe the potential for Tpto activate platelets and to recognize specific platelet secretions.

Methods Viable $T p$ and platelets were co-incubated and platelet activation was compared relative to pre-activated (ACT) and resting (REST) platelets. Platelet activation was measured by assessing the median fluorescence intensity (MFI) of the platelet activation receptor CD41a via flow cytometry and fibrin clot formation (a downstream effect of platelet activation) via plate-based assays. A capillary tube chemotaxis assay quantified $T p$ migration towards specific secretions from activated platelets compared to inactive platelets.

Results Tp co-incubated with platelets and ACT platelets expressed comparable levels of CD41a, with an almost $150 \%$ greater CD41a expression level compared to that seen with REST platelets $(\mathrm{P}=0.0118)$. Tp co-incubated with platelets and ACT platelets produced similar levels of clotting, at a level that was significantly higher than for REST platelets ( $P$ $=0.0221)$. Tp migration towards the secretions of activated platelets was over 2 -fold higher than migration towards inactive platelets $(\mathrm{P}=0.0030)$.

Conclusion $T p$ activates platelets and migrates towards the secretions of activated platelets. Prior investigations have established that platelet activation, and subsequent secretion, enhances the permeability of endothelial cells lining the bloodstream. Taken together, these findings suggest the treponeme-platelet interaction may promote $T p$ entry and exit from the bloodstream and aid in $T p$ spread throughout the body.

Disclosure No significant relationships.

\section{P730 THE DEMOGRAPHY OF CONGENITAL SYPHILIS ELIMINATION IN THE UNITED STATES}

Najjuwah Walden*. Washington University in St. Louis, St. Louis, USA

\subsection{6/sextrans-2019-sti.791}

Background The absence of reported congenital syphilis cases in 16 states and territories across the United States of America in 2017 may be attributable to changes in prenatal care utilization within these states from 2013-2017. In order to determine whether there is a relationship between rates of reported congenital syphilis cases and prenatal care utilization in the United States, we assessed changes in 16 states and territories with a congenital syphilis rate of 0.0 per 100,000 per live births in 2017. We determined the association between rates of congenital syphilis and utilization from 2013 to 2017, and explored the association between utilization and changes to state, territorial, and local mandates on congenital syphilis. Methods We retrieved vital statistic data on congenital syphilis infections; prenatal care; and patient demographics. We retrieved public reports of mandate changes from state, territorial, and local public health agencies. Vital statistic data were collected by the United States Centers for Disease Control and Prevention National Vital Statistic System from 2013 to 2017. Cases included births of Non-Hispanic and Hispanic postpartum patients, who received congenital syphilis testing after live births in 2013, 2014, 2015, 2016, and 2017. We analyzed the incidence rate ratio (IRR) of congenital syphilis cases and prenatal care utilization. We then analyzed mandates to assess whether any changes to congenital syphilis screening could explain suppression or elimination of syphilis.

Results Increases in prenatal care utilization led to a decrease in congenital syphilis rates, and associations were stronger within states with an overrepresentation of Non-Hispanic white patients.

Conclusion While mandates may explain suppression or elimination of congenital syphilis, additional research is needed to determine whether the reduction is predicted by patient demographics, rather than mandated screening.

Disclosure No significant relationships.

\section{P731 PREVALENCE OF SELF-REPORTED SYPHILIS AMONG BRAZILIAN YOUNG ADULTS: FINDINGS FROM A NATIONWIDE SURVEY}

${ }^{1}$ Natalia Kops*, ${ }^{1}$ Marina Bessel, ${ }^{2}$ Adele Benzaken, ${ }^{1}$ Eliana Wendland. ${ }^{1}$ Hospital Moinhos de Vento, Porto Alegre, Brazil; ${ }^{2}$ Fundação de Medicina Tropical Doutor Heitor Vieira Dourado, Manaus, Brazil

\subsection{6/sextrans-2019-sti.792}

Background The number of cases of acquired syphilis are increasing in many countries. In Brazil, a previous official report based on compulsory notifications showed a sustained increase from 2.0 to 58.1 cases per 100,000 inhabitants over the last six years (2010-2017). We aimed to evaluate the prevalence of self-reported syphilis among women and men aged 16 to 25 years who use the public health system in Brazil.

Methods This is a cross-sectional, nationwide, multicenter study with 8,581 sexually active young adults recruited from 119 primary care units in all 26 Brazilian capitals and the Federal District. Data on sociodemographic and sexual behavior characteristics were obtained by face-to-face interviews. To evaluate syphilis, we asked the participants if they had ever had syphilis during their lives (current or in the past). We weighted the measures by population size in each capital and by sex.

Results Of all participants (49.17\% women), 8,076 provided information about syphilis, and 224 (2.86\%, 95\%CI2.29\%$3.43 \%)$ reported having the disease. The prevalence was similar between genders $(p=0.240)$, with frequencies of $3.20 \%$ for men and $2.54 \%$ for women. The participants who selfreported syphilis were older [22.35 years $(22.01-22.70)]$ than those without the disease [21.38 years (21.32-21.44); $\mathrm{p}<0.001]$ and had earlier sexual intercourse [14.81 years (14.53-15.09) vs. 15.22 years (15.17-15.27); $p=0.006]$. Syphilis was not associated with income and skin color/race, but it was significantly associated with educational level. Illiterate and elementary students had higher prevalence than other 